\title{
The role of the tumor suppressor RUNX3 in giant cell tumor of the bone
}

\author{
YA-XIN HAN and DE-YONG LIANG \\ Department of Orthopaedics, First Affiliated Hospital of China Medical University, Shenyang 110001, P.R. China
}

Received August 20, 2011; Accepted October 5, 2011

DOI: 10.3892/ijo.2011.1249

\begin{abstract}
RUNX3 is a tumor suppressor gene localized in 1p36. In various human tumors, the region is frequently inactivated through hypermethylation, histone modulation and other processes. Recent studies have suggested that loss of RUNX3 expression is involved in stomach, colon and breast cancer. However, the relationship between RUNX3 expression and giant cell tumor of the bone (GCTB) remains elusive. The aim of our study was to elucidate the roles of RUNX3 expression in carcinogenesis and progression of giant cell tumor of the bone. The levels of RUNX3 mRNA and protein were evaluated in human GCTB specimens and cell lines. To assess RUNX3 methylation we employed methylation-specific polymerase chain reaction using GCTB specimens and cell lines. In addition, to examine the roles of RUNX3 in giant cell tumor of the bone, GCTB cells were transfected with pcDNA3.1-RUNX3 (RUNX3 was cloned into the pcDNA3.1 plasmid). Flow cytometry (FCM) was used to analyze the apoptosis and cell cycle. The mobility of cells was tested by transwell migration assay. The expression rates of RUNX3 in patients with GCTB were significanly lower than normal bone tissues. Thirty of 47 human cancer specimens exhibited suppression $(\mathrm{P}<0.05)$. Down-regulation of RUNX3 mRNA in the same GCTB cell lines was associated with RUNX3 DNA methylation. In in vitro experiments, exogenous expression of RUNX3 strongly inhibited cell growth in GCTB by MTT $(\mathrm{P}<0.05)$, induced apoptosis as evidenced by Annexin V-FITC and increased $\mathrm{G}_{1}$ phase ratio by $\mathrm{PI}(\mathrm{P}<0.05)$. Transwell migration assay showed that less RUNX3 positive cells migrated to the lower side of the membrane than negative ones $(\mathrm{P}<0.05)$. These results show that RUNX3 is a tumor suppressor in GCTB. RUNX3 DNA methylation may be the molecular basis for its lower expression. These data may be applied in GCTB for diagnostics and therapeutics.
\end{abstract}

Correspondence to: Dr De-Yong Liang, Department of Orthopaedics, First Affiliated Hospital of China Medical University, Shenyang 110001, P.R. China

E-mail: doctor_yaxin@163.com

Key words: giant cell tumor of bone, RUNX3, methylation, apoptosis, cell cycle

\section{Introduction}

Giant cell tumor of bone (GCTB) is a high leading cause of cancer-related deaths worldwide, and poor prognosis and high mortality continue to be problems with this cancer $(1,2)$. Generally, young adults aged 20 and 40 years tend to suffer GCTB. It can cause osteolysis and rupture at the ends of the femur and the tibia (3-5). Despite its prevalence, the mechanisms of pathogenesis and development of GCTB remains unclear. In recent years, multiple gene changes have long been associated with GCTB. The Runt-related (RUNX) family of transcription factors, RUNX1, RUNX2 and RUNX3, can generate heterodimeric transcription regulators (6). All these members play an important role in carcinogenesis and progression of malignancies (7-10). Especially, RUNX3 as a new candidate tumor suppressor gene has been implicated in multiple cancers, such as liver, stomach, prostate, colon and breast cancer (11-15). Furthermore, promoter hypermethylation has been demonstrated as a cause of RUNX3 protein lower expression in prostate, breast and bladder cancer $(13,15,16)$. But very interesting, Tsunematsu et al used siRNA to knock down RUNX3 in a head and neck cancer-derived cell lines. The results showed the cell growth was inhibited, suggesting that RUNX3 functions as an oncogene (17). To our knowledge, no studies have reported on the effects of RUNX3 on GCTB until now. We hope to elucidate the roles of RUNX3 in carcinogenesis and progression of GCTB.

\section{Materials and methods}

Cell lines and GCTB patient specimens. The cellular elements of GCTB include both osteoclast-like giant cells and osteoblast-like stromal cells (18). Mak et al $(19,20)$ established primary cell cultures of GCTB stromal tumor cells from fresh GCTB tissue. According to their method, we got osteoclastlike giant cells from the specimens with razor blades. The cell lines were named after $\mathrm{O} 1, \mathrm{O} 2, \mathrm{O} 3, \mathrm{O} 4, \mathrm{O} 5$ and cultured in DMEM (Hyclone, USA) supplemented with 10\% FBS and antibiotics (100 U/ml penicillin and $100 \mu \mathrm{g} / \mathrm{ml}$ streptomycin) in a $37^{\circ} \mathrm{C}$ humidified incubator with $95 \%$ air, $5 \% \mathrm{CO}_{2}$. GCTB tissue specimens were derived from 47 patients no chemotherapy or radiotherapy before the resection at the department of orthopaedics, First Affiliated Hospital of China Medical University between July 2006 and January 2010. According to Campanacci et al (21), the patient specimens were classified by 
Grade I (22), Grade II (13) and Grade III (12). The patients all approved the use of tumor tissues for clinical research and our university ethics committee approved the research protocols.

RNA isolation and reverse transcriptase-polymerase chain reaction $(R T-P C R)$. TotalRNA was isolated using a RNeasy mini kit (BioMed, China). First strand cDNA was reverse transcribed with $1 \mu \mathrm{g}$ of total RNA, using Takara Reverse Transcription Kit (Takara, Japan) and oligo(dT) 15 primers (Takara). The RUNX3 primers were 5'-GAGTTTCACCCTGACCATCACTGTG-3' (sense) and 5'-GCCCATCACTGGTCTTGAAGGTTGT-3' (antisense). The housekeeping gene, GAPDH was used as an internal control for normalization of the results. The GAPDH primers were 5'-AGAAGGCTGGGGCTCATTTG-3' (sense) and 5'-AGGGGCCATCCACAGTCTTC-3' (antisense). PCR amplification of cDNA was performed in $20 \mu \mathrm{l}$ mixtures. Finally, amplicons were electrophoresed in $2 \%$ agarose gel with ethidium bromide and visualized under UV illumination.

Methylation-specific PCR (MSP). Genomic DNA was extracted from GCTB specimens and cell lines using a TissueGen DNA Kit (CW Biotechnology, China). Genomic DNA $(2 \mu \mathrm{g})$ was denatured with $0.2 \mathrm{M} \mathrm{NaOH}$. Then, $10 \mathrm{mM}$ hydroquinone (Sigma, USA) and 3 M sodium-bisulfite (Sigma) were added. The solution was incubated at $55^{\circ} \mathrm{C}$ for $16 \mathrm{~h}$. DNA samples were then purified using a Wizard DNA Purification Resin (Promega, USA). In this procedure unmethylated (but not methylated) cytosines can convert to uracil, which is then converted to thymidine during subsequent PCR to give sequence differences between methylated and unmethylated DNA. The modified DNA was used as a template both for MSP and USP. The primer sequences for the methylated RUNX3 gene were 5'-TTACGAGGGGCGGTCGTACGCGGG-3' (sense) and 5'-AAAACGACCGACGCGAACGCCTCC-3' (antisense), and for the unmethylated allele were 5'-TTATGAG GGGTGGTTGTATGTGGG-3' (sense) and 5'-AAAACAA CCAACACAAACACCTCC-3' (antisense). The PCR products were separated in $2 \%$ agarose gel with ethidium bromide and visualized under UV illumination. Cells were incubated with medium containing $10 \mu \mathrm{M}$ 5-aza-2'-deoxycytidine (5-aza-dC) (Sigma) for $48 \mathrm{~h}$. Then we isolated DNA and carried out MSP as described above.

Western blot analysis. GCTB specimens and cell lines were lysed in $20 \mathrm{mM}$ Tris- $\mathrm{HCl}$ buffer ( $\mathrm{pH} 7.4$ ) containing $150 \mathrm{mM}$ $\mathrm{NaCl}, 2 \mathrm{mM}$ EDTA, $1 \%$ Nonidet P-40, $50 \mathrm{mM} \mathrm{NaF}, 1 \mathrm{mM}$ $\mathrm{Na}_{3} \mathrm{VO}_{4}, 1 \mathrm{mM} \mathrm{Na} \mathrm{MoO}_{4}, 10 \mu \mathrm{M}$ aprotinin and $10 \mu \mathrm{M}$ leupeptin. The solution was centrifuged at $15000 \mathrm{~g}$ for $5 \mathrm{~min}$, and the supernatant was removed. Equal amounts of cell lysate protein extracts were fractionated by sodium dodecyl sulfate polyacrylamide gel electrophoresis and transferred to an $\mathrm{NC}$ membrane. The membranes were incubated with anti-RUNX3 (Sigma) and $\beta$-actin antibodies (Sigma) for $24 \mathrm{~h}$ at $4^{\circ} \mathrm{C}$, respectively, and then with peroxidase-conjugated secondary antibodies for $2 \mathrm{~h}$ at room temperature. The blotted proteins were detected using an ECL Kit (Takara).

Plasmids. The cDNA of RUNX3 was obtained by RT-PCR as described above. The cDNA fragment was digested with $E c o$ RI and $X h o l$ and inserted into a pcDNA3.1-myc vector.
Stable cell lines. Cells were transfected using Lipofectamine ${ }^{\mathrm{TM}}$ 2000 (Invitrogen, USA) according to the manufacturer's instructions. RUNX3-restored cells were obtained by transfecting with pcDNA3.1-RUNX3. G418-resistant clones were selected using G418 (500 ng/ $\mu$ l). The RUNX3 positive cell lines were named after R1, R2, R3, R4, R5.

Cell growth inhibition assays (MTT assay). We selected R4 to represent RUNX3 positive cells and O2 to represent RUNX3 negative cells. $\mathrm{R} 4$ and $\mathrm{O} 2$ were seeded in quadruplex in 96-well dishes at a density of 1500 cells/well, allowed to attach overnight. 3-(4,5-Dimethylthiazol-2-yl)-2,5-diphenyltetrazolium bromide (MTT) solution was added (final concentration $0.5 \mathrm{mg} / \mathrm{ml}$ ) (Sigma), for $4 \mathrm{~h}$. Absorbance were measured at $550-560 \mathrm{~nm}$.

Cell cycle analysis. $\mathrm{R} 4$ and $\mathrm{O} 2$ were collected and centrifuged at $1500 \mathrm{~g}$ for $5 \mathrm{~min}$ and the pellet resuspended in $100 \mu \mathrm{l}$ PBS at a density of $1 \times 10^{6}$ cells $/ \mathrm{ml}$. Added $900 \mu 170 \%$ cold ethanol to the mixture for $1 \mathrm{~h}$ on ice. Cells were collected at $1500 \mathrm{~g}$ for $5 \mathrm{~min}$. Then the pellet was resuspended in $100 \mu \mathrm{l}$ PBS containing RNaseA (final concentration $0.2 \mathrm{mg} / \mathrm{ml}$ ) (Sigma) and leaving at room temperature for $30 \mathrm{~min}$. Cells were recovered once more and the pellets resuspended in 350 of $5 \mu 1$ propidium iodide (PI) (Keygene, China) in PBS before analyzed by a FACSCalibur machine (Model FACSC420, USA).

Cell apoptosis assay. Apoptosis was determined using an apoptosis detection kit (Keygene). Briefly, R4 and O2 were collected, washed twice in ice-cold PBS and then resuspended in binding buffer at a density of $1 \times 10^{6}$ cells/ $\mathrm{ml}$. Cells were incubated simultaneously with fluoresceinlabeled Annexin V and propidium iodide (PI) for $20 \mathrm{~min}$. Then the mixture was analyzed by a FACSCalibur machine. Annexin V-FITC generated signals were detected with an FITC signal detector (FL1, $525 \mathrm{~nm}$ ). PI signals were monitored using a detector reserved for phycoerythrin emission (FL2, $575 \mathrm{~nm}$ ). Data were analyzed using CellQuest software from BD.

Transwell migration assay. The migration assay was performed using transwell cell culture chambers $(8 \mu \mathrm{M}$ pore size polycarbonate membrane; Costar). Cells were resuspended in DMEM with $0.1 \%$ FBS to a concentration of $3 \times 10^{5}$ cells $/ \mathrm{ml}$. The upper chamber was loaded with $100 \mu \mathrm{l}$ of cell suspension and the lower chamber was loaded with $600 \mu \mathrm{l}$ of DMEM with $10 \%$ FBS. After incubation for $24 \mathrm{~h}$ at $37^{\circ} \mathrm{C}$ with $5 \% \mathrm{CO}_{2}$, the filter was fixed with $4 \%$ paraformaldehyde and stained with crystal violet (CV). The cells on the upper side of the filter were wiped off with a cotton swab. The cells migrated to the undersurface of the membrane were counted under microscope. Ten microscopic fields (x400) were randomly selected to count cells. Each assay was done in triplicate.

Statistical analysis. All numerical data were expressed as means $\pm \mathrm{SD}$. Differences among the mean values were evaluated using Student's t-test. All statistical analyses were conducted by SPSS 11.0 (SPSS Inc., USA). P-values $<0.05$ were considered statistically significant. 
Table I. RUNX3 proteins expression in GCTB specimens.

\begin{tabular}{lrrrr}
\hline & & \multicolumn{3}{c}{ RUNX3 expression } \\
\cline { 3 - 5 } Lesion of grades & $\mathrm{N}$ & - & + & NR (\%) \\
\hline Grade I & 22 & 17 & 5 & 77 \\
Grade II & 13 & 8 & 5 & 61.5 \\
Grade III & 12 & 9 & 3 & 75 \\
\hline
\end{tabular}

NR, negative rate.
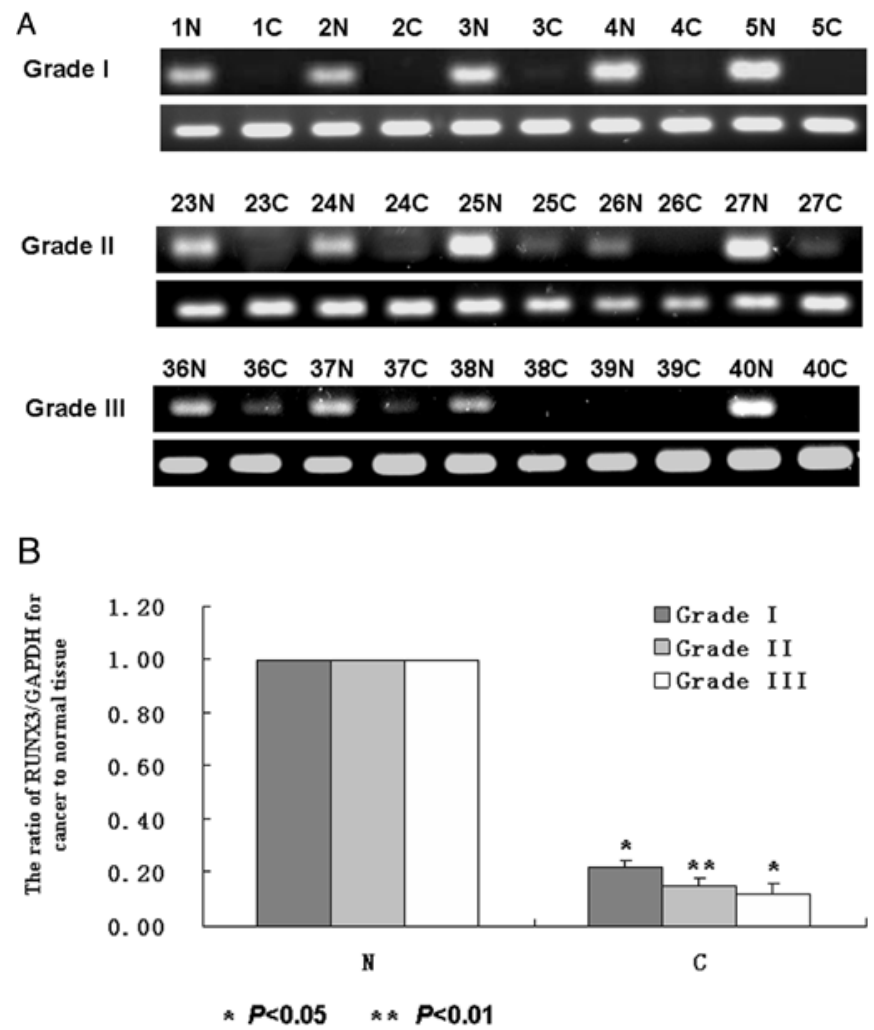

C

\begin{tabular}{lllllllllll}
01 & 02 & 03 & 04 & 05 & R1 & R2 & R3 & R4 & R5 \\
\hline & & & & & & & & & &
\end{tabular}

Figure 1. The level of RUNX3 mRNA was measured in specimens using RT-PCR. GADPH was used as an internal control. (A) The level of RUNX3 mRNA was detected in specimens according to the lesion grades. N, normal; C, cancer. (B) The level of RUNX3 mRNA was lower in GCTB than matched normal tissues $(\mathrm{P}<0.05)$. N, normal; $\mathrm{C}$, cancer. (C) The level of RUNX3 mRNA was detected in osteoclast-like giant cells $\mathrm{O} 1, \mathrm{O} 2, \mathrm{O} 3, \mathrm{O} 4, \mathrm{O} 5$ and transfected cells R1, R2, R3, R4, R5.

\section{Results}

The level of RUNX3 mRNA and protein was evaluated in 47 human GCTB specimens and cell lines. Western blot analysis was carried out to investigate the protein status of RUNX3 in GCTB specimens (Fig. 2A). We analyzed the specimens
A

Grade I

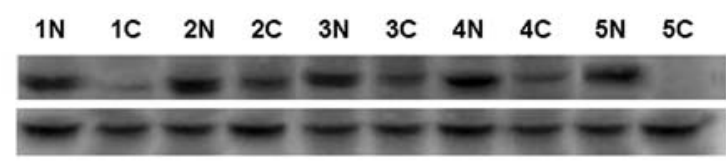

Grade II
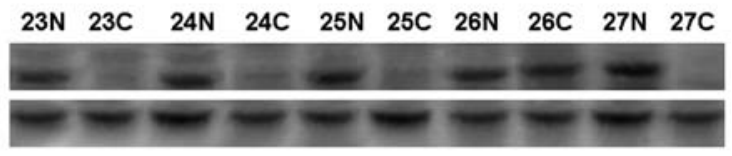

$\begin{array}{llllllllll}36 \mathrm{~N} & 36 \mathrm{C} & 37 \mathrm{~N} & 37 \mathrm{C} & 38 \mathrm{~N} & 38 \mathrm{C} & 39 \mathrm{~N} & 39 \mathrm{C} & 40 \mathrm{~N} & 40 \mathrm{C}\end{array}$

Grade III

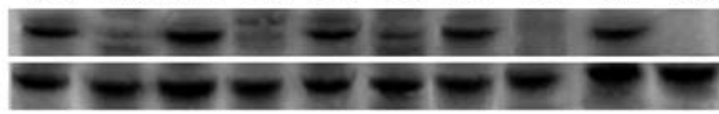

B

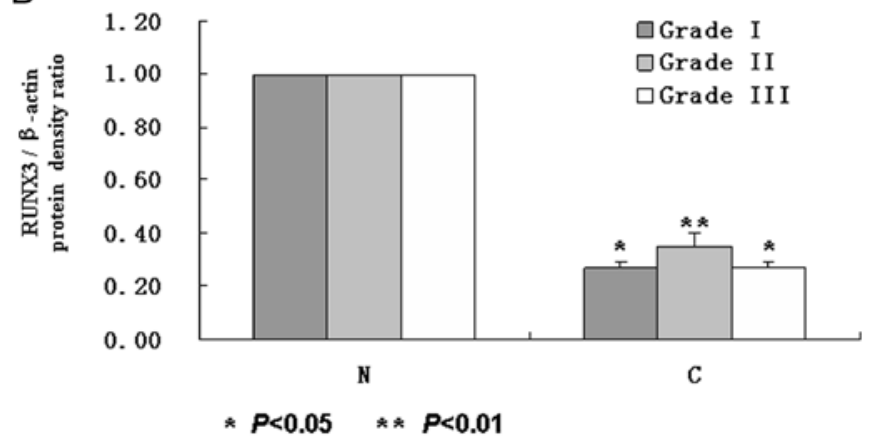

C

$\begin{array}{lllllllllll}01 & 02 & O 3 & O 4 & O 5 & R 1 & R 2 & R 3 & R 4 & R 5\end{array}$

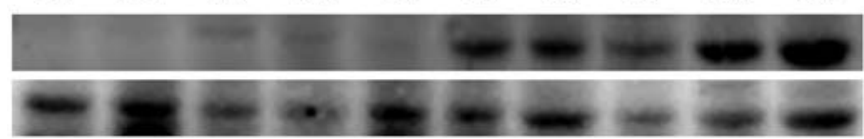

Figure 2. Western blotting for RUNX3 proteins in specimens and cell lines. $\beta$-actin was used as an internal control. (A) Expression of RUNX3 proteins was detected in specimens according to the lesion grades. $\mathrm{N}$, normal; $\mathrm{C}$, cancer. (B) RUNX3 proteins expression level was lower in GCTB than matched normal tissues $(\mathrm{P}<0.05)$. N, normal tissue; $\mathrm{C}$, cancer. (C) Expression of RUNX3 was detected in osteoclast-like giant cells O1-5 and transfected cells R1-5.

according to Grade I (22), Grade II (13) and Grade III (12), respectively. As shown in the results, the level of RUNX3 protein exhibited lower than normal tissue but not related with the grade of lesion $(\mathrm{P}<0.05$, Fig. $2 \mathrm{~B})$. We can see that RUNX3 expression was absent in 13 of 22 Grade I (59\%), 8 of 13 Grade II (61.5\%) and 9 of 12 Grade III (75\%) in the Table I. To examine the relationship between the level of RUNX3 protein and the level of RUNX3 transcription, RT-PCR of RUNX3 mRNA was carried out in GCTB specimens. The results showed that the level of RUNX3 mRNA was also lower than normal tissue and coincident with the level of protein $(\mathrm{P}<0.05$, Fig. 1).

Down-regulation of RUNX3 mRNA in GCTB cell lines but not in specimens correlate significantly with RUNX3 promoter methylation. Forty tumor samples and GCTB cell lines were examined for RUNX3 methylation by MSP. All tumor samples and cell lines had unmethylated alleles. Representative examples are illustrated in Fig. 3. We found a correlation between CpG island RUNX3 promoter methylation and down-regulated RUNX3 mRNA levels in GCTB cell lines (Fig. 3B) but not 
A

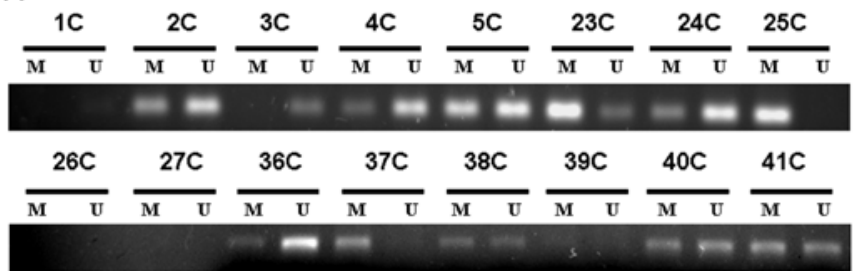

B

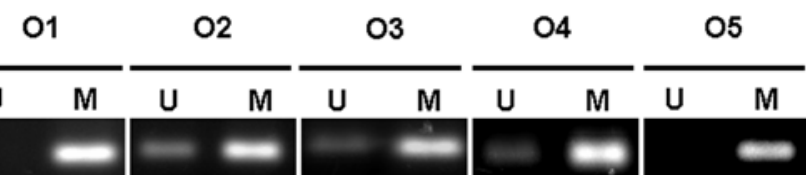

C

M

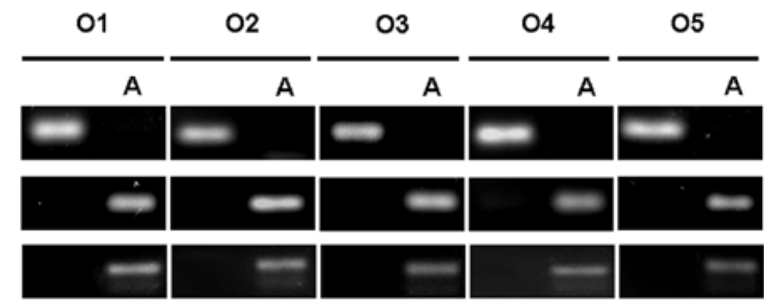

Figure 3. (A) Methylation-specific PCR (MSP) analysis of the CpG island methylation status of the RUNX3 promoter region in specimens. PCR products specific for unmethylated (U) and methylated (M) CpG sites were analysed in 2\% agarose gel. (B) MSP was used to analyze the CpG island methylation status in osteoclast-like giant cells. (C) After treated with 5-aza$\mathrm{dC}$, methylation status was analyzed by MSP and the RUNX3 mRNA was restored.

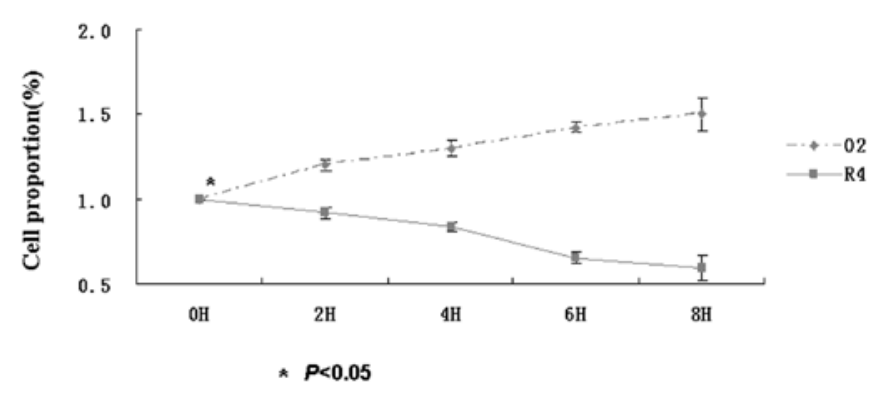

Figure 4. The percentages of growth inhibition of RUNX3 on R4 were determined by MTT in comparison with O2. RUNX3 showed an inhibitory effect on cell growth $(\mathrm{P}<0.05)$.

tissues (Fig. 3A). After the cells were treated with 5-aza-dC, we found that the RUNX3 mRNA was restored and methylation disappeared (Fig. 3C).

The biological functions of excess RUNX3 on GCTB cells. To further investigate the functions of RUNX3, we constructed pcDNA3.1-RUNX3. Full-length RUNX3 was exogenously expressed in GCTB cell lines. As shown in the Figs. 1C and $2 \mathrm{C}$, the level of protein and mRNA in GCTB cell lines exhibited lower than transfected cells. MTT assay showed that the proliferation rates of RUNX3 positive cells (R4) were decreased compared with negative cells $(\mathrm{O} 2)(\mathrm{P}<0.05$, Fig. 4). As shown in FCM, the ratio of apoptosis (Fig. 5) and $\mathrm{G}_{1}$ phase
A

02
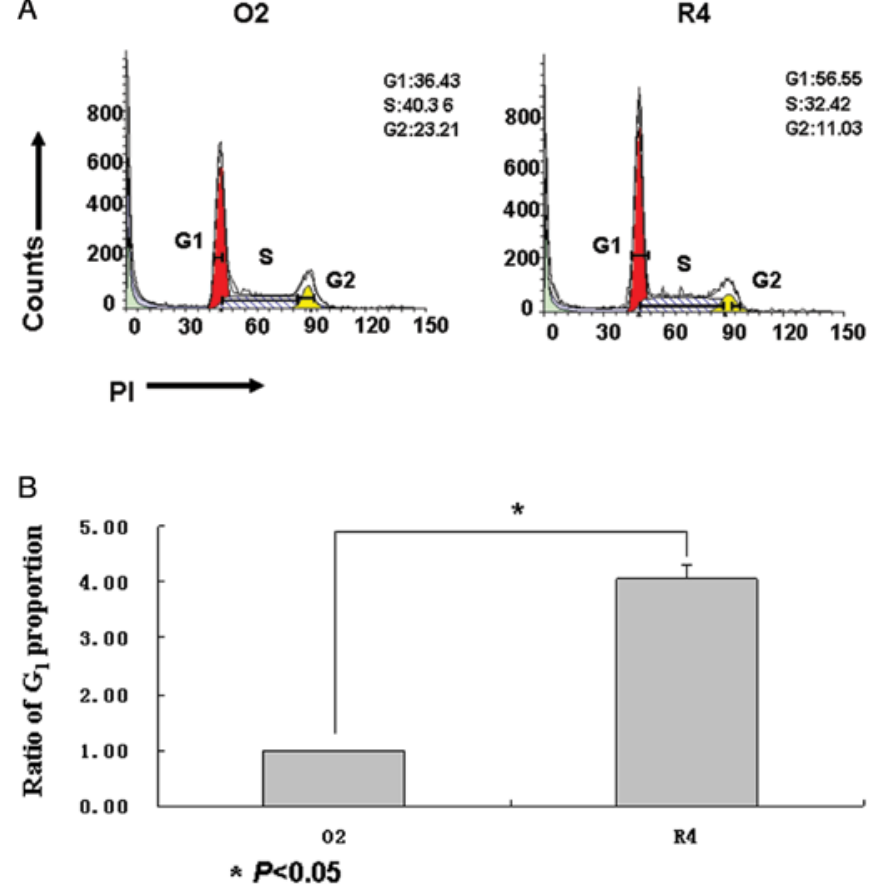

Figure 5. (A) Propidium iodide staining shows changes in the cell cycle. A marked increase in the $\mathrm{G}_{1}$ fraction of $\mathrm{R} 4$ compared with $\mathrm{O} 2$, as well as the appearance of the $G_{1}$ fraction. (B) The histogram shows the ratio of $G_{1}$ was increased in $\mathrm{R} 4$ in comparison with $\mathrm{O} 2(\mathrm{P}<0.05)$.

A

02

R4
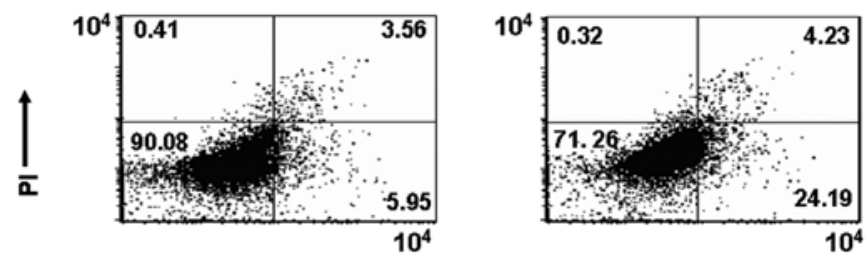

Annexin VFITC

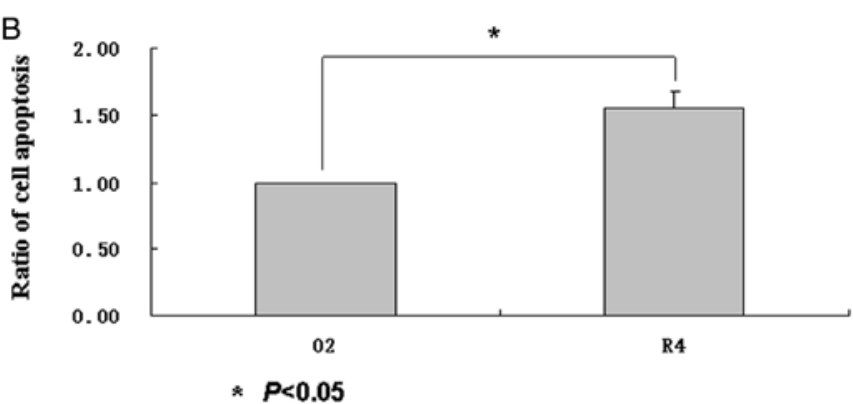

Figure 6. (A) Apoptosis ratio of $\mathrm{R} 4$ and $\mathrm{O} 2$ was analyzed by double stained with Annexin V/PI. (B) The histogram shows the apoptosis ratio of R4 was $>\mathrm{O} 2(\mathrm{P}<0.05)$.

(Fig. 6) were increased in RUNX3 positive cells $(\mathrm{P}<0.05)$. In order to have a better understanding of the changes of cell phenotype, we used transwell migration assay to examine the mobility of RUNX3 positive cells. It showed that less RUNX3- 
A
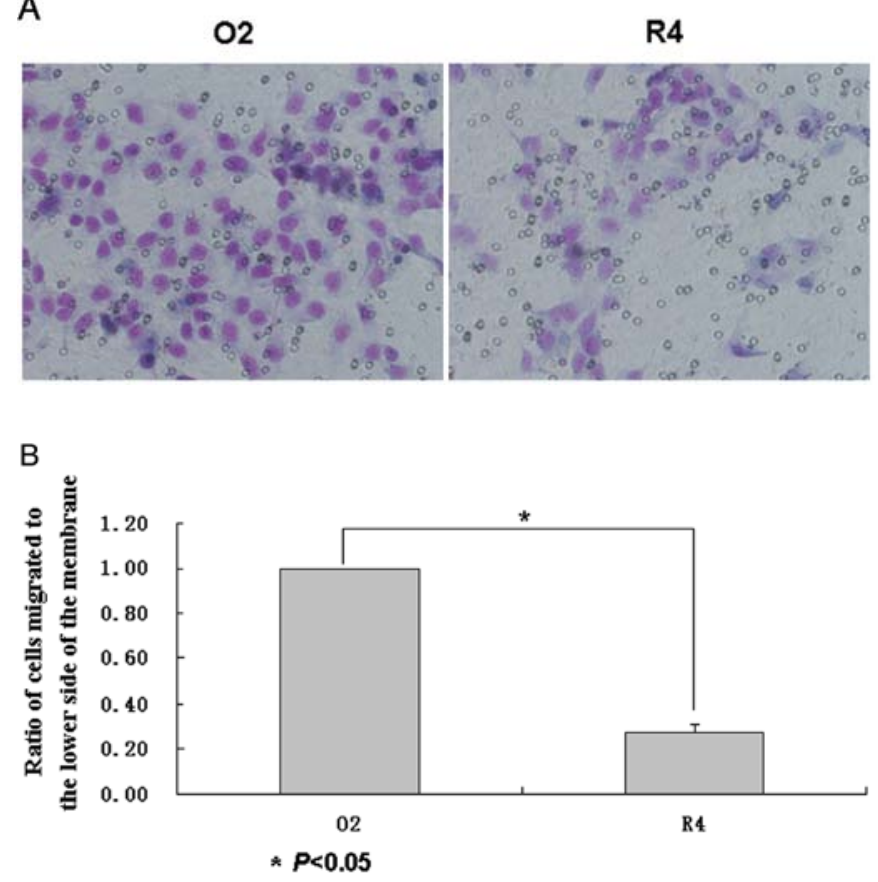

Figure 7. (A) Migrated cells found on the bottom side of the membrane were fixed, and stained. (B) Numbers of migrated cells on the membrane bottom were counted. Cell migration is represented as a percentage, normalized to O2. RUNX3 played a negative role in $\mathrm{R} 4$ migration $(\mathrm{P}<0.05)$.

positive cells migrated to the lower side of the membrane than negative ones $(\mathrm{P}<0.05$, Fig. 7$)$.

\section{Discussion}

RUNX3 as a tumor suppressor gene was first identified in gastric cancer (12). In recent studies, the role of RUNX3 inactivation has been investigated not only in human cancers (11-15) but also in preneoplastic lesions such as chronic gastritis (22), viral infections of liver (22), intestinal metaplasia (22), Barrett's oesophagus (23), colorectal polyps (24), atypical adenomatous hyperplasia of lung (25), prostatic intraepithelial neoplasia (13). In this study, we first analyzed the expression of RUNX3 gene in GCTB specimens and cell lines. We show representative results in Fig. 2. The results were coincident with the level of the RUNX3 mRNA. But there is no difference among the lesion of grades. The results indicated that loss of RUNX3 may associate with carcinogenesis but not with aggravation of GCTB. Then we analyzed the methylation status of the RUNX3 gene. Aberrant methylation of normally unmethylated $\mathrm{CpG}$ rich areas (or islands) in, or near the promoter region has been associated with transcriptional inactivation of tumor suppressor in many cancers (26-28). In many cancer types, RUNX3 protein lower expression has been correlated with promoter hypermethylation $(13,15,16)$. However, we did not detect RUNX3 promoter methylation in GCTB specimens. As shown in Fig. 3A, the data indicated that down-regulation of RUNX3 protein may not be based on the methylation of the promoter. The molecular basis for RUNX3 protein lower expression is still unclear. Further studies are planned to solve this problem. However, we found RUNX3 promoter methylation in GCTB cell lines, and that the RUNX3 promoter methylation was related with down-regulated RUNX3 mRNA levels. As shown in Fig. 3B and C, after treatment with 5-aza-dC, not only RUNX3 promoter methylation disappeared but also the expression RUNX3 mRNA was restored. Methylation of promoter may be a molecular basis for RUNX3 protein lower expression in GCTB cell lines.

In previous studies, Chi et al (29) showed that RUNX3 overexpression inhibits proliferation and induces cell cycle arrest in gastric cancer cell lines. Lee et al (30) and Yamamura et al (31), respectively reported similar conclusion in colorectal cancer cell lines and in neuroblastoma cell lines. Similarly, in our results, RUNX3 positive cells also demonstrated proliferation inhibitory effects and increased $G_{1}$ phase ratio. RUNX3 appears to play a significant role in GCTB to inhibit cell growth. RUNX3 has been shown to mediate cell growth by regulating apoptosis $(17,32,33)$. In our studies, apoptosis was determined by Annexin V-FITC and PI double staining with flow cytometry. As shown in Fig. 6, RUNX3 can induce apoptosis in GCTB. To our knowledge, no related studies have identified whether RUNX3 contribute to tumor metastasis. Since metastasis depends on mobility of cancer cells, we detected the effects of RUNX3 on GCTB cell lines using transwell migration assay. The result of assay showed that RUNX3 can inhibit migration of GCTB cells. It indicated that RUNX3 may interact with some mobility related protein to reduce movement of cancer cells. That may provide a new direction to our research.

In summary, this study first identified RUNX3 as a novel suppressor gene in GCTB. These findings provided a new insight into the role of RUNX3 in the process of carcinogenesis and development in GCTB and helped to improve the diagnosis and therapeutics of GCTB. However, the signal pathway of RUNX3 in GCTB remains unclear. In further studies, we will investigate the upstream and downstream protein of RUNX3 in order to determine the related signal pathways.

\section{Acknowledgements}

We thank Dr Nan Xu for pcDNA3.1 plasmid and Dr Miao Zhang for technical assistance.

\section{References}

1. Mendenhall WM, Zlotecki RA, Scarborough MT, et al: Giant cell tumor of bone. Am J Clin Oncol 29: 96-99, 2006.

2. Parkin DM: Global cancer statistics in the year. Lancet Oncol 2: 533-543, 2000.

3. Lee CH, Espinosa I, Jensen $\mathrm{KC}$, et al: Gene expression profiling identifies p63 as a diagnostic marker for giant cell tumor of the bone. Mod Pathol 21: 531-539, 2008.

4. Khalil ESA, Younis A, Aziz SA, et al: Surgical management for giant cell tumor of bones. J Egypt Natl Canc Inst 16: 145-152, 2004.

5. Turcotte RE: Giant cell tumor of bone. Orthop Clin North Am 35: 35-51, 2006.

6. Ogawa E, Maruyama M, Kagoshima H, et al: PEBP2/PEA2 represents a family of transcription factors homologous to the products of the Drosophila runt gene and the human AML1 gene. Proc Natl Acad Sci USA 90: 6859-6863, 1993.

7. Lund AH and van Lohuizen M: RUNX: a trilogy of cancer genes. Cancer Cell 1: 213-215, 2002.

8. Otto F, Kanegane H, Mundlos S, et al: Mutations in the RUNX2 gene in patients with cleidocranial dysplasia. Hum Mutat 19: 209-216, 2002. 
9. Blyth K, Cameron ER, Neil JC, et al: The RUNX genes: gain or loss of function in cancer. Nat Rev Cancer 5: 376-387, 2005.

10. Kohu K, Sato T, Ohno S, et al: Overexpression of the Runx3 transcription factor increases the proportion of mature thymocytes of the CD8 single-positive lineage. J Immunol 174: 2627-2636, 2005.

11. Xiao WH and Liu WW: Hemizygous deletion and hypermethylation of RUNX3 gene in hepatocellular carcinoma. World J Gastroenterol 10: 376-380, 2004.

12. Li QL, Ito K, Sakakura C, et al: Causal relationship between the loss of RUNX3 expression and gastric cancer. Cell 109: 113-124, 2002.

13. Kang GH, Lee S, Lee HJ, et al: Aberrant $\mathrm{CpG}$ island hypermethylation of multiple genes in prostate cancer and prostatic intraepithelial neoplasia. J Pathol 202: 233-240, 2004.

14. Lee CW, Ito K and Ito Y: Role of RUNX3 in bone morphogenetic protein signaling in colorectal cancer. Cancer Res 70: 4243-4252, 2010.

15. Lau QC, Raja E, Salto-Tellez M, et al: RUNX3 is frequently inactivated by dual mechanisms of protein mislocalization and promoter hypermethylation in breast cancer. Cancer Res 66: 6512-6520, 2006.

16. Kim WJ, Kim EJ, Jeong P, et al: RUNX3 inactivation by point mutations and aberrant DNA methylation in bladder tumors. Cancer Res 65: 9347-9354, 2005.

17. Tsunematsu T, Kudo Y, Iizuka S, et al: RUNX3 has an oncogenic role in head and neck cancer. PLoS One 4: e5892, 2009.

18. Ghert M, Simunovic N, Cowan RW, et al: Properties of the stromal cell in giant cell tumor of bone. Clin Orthop Relat Res 459: 8-13, 2007.

19. Mak IW, Turcotte RE, Popovic S, et al: AP-1 as a regulator of MMP-13 in the stromal cell of giant cell tumor of bone. Biochem Res Int 2011: 164197, 2011.

20. Mak IW, Cowan RW, Popovic S, et al: Upregulation of MMP-13 via Runx 2 in the stromal cell of giant cell tumor of bone. Bone 45: 377-386, 2009.

21. Campanacci M, Baldini N, Boriani S, et al: Giant-cell tumor of bone. J Bone Joint Surg Am 69: 106-114, 1987.

22. Kim TY, Lee HJ, Hwang KS, et al: Methylation of RUNX3 in various types of human cancers and premalignant stages of gastric carcinoma. Lab Invest 84: 479-484, 2004.
23. Schulmann K, Sterian A, Berki A, et al: Inactivation of p16, RUNX3, and HPP1 occurs early in Barrett's associated neoplastic progression and predicts progression risk. Oncogene 24: 4138-4148, 2005

24. Subramaniam MM, Chan JY, Soong R, et al: RUNX3 inactivation in colorectal polyps arising through different pathways of colonic carcinogenesis. Am J Gastroenterol 104: 426-436, 2009.

25. Licchesi JD, Westra WH, Hooker CM, et al: Epigenetic alteration of Wnt pathway antagonists in progressive glandular neoplasia of the lung. Carcinogenesis 29: 895-904, 2008.

26. Merlo A, Herman JG, Mao L, et al: 5-CpG island methylation is associated with transcriptional silencing of the tumour suppressor p16/CDKN2/MTS1 in human cancers. Nat Med 1: 686-692, 1995.

27. Zochbauer-Muller S, Fong KM, et al: Aberrant promoter methylation of multiple genes in non-small cell lung cancers. Cancer Res 61: 249-255, 2001

28. Yanagawa N, Tamura G, Oizumi H, et al: Promoter hypermethylation of tumor suppressor and tumor-related genes in non-small cell lung cancers. Cancer Sci 94: 589-592, 2003.

29. Chi XZ, Yang JO, Lee KY, et al: RUNX3 suppresses gastric epithelial cell growth by inducing $\mathrm{p} 21^{\mathrm{WAF} / / \mathrm{Cip} 1}$ expression in cooperation with transforming growth factor $\beta$-activated SMAD. Mol Cell Biol 25: 8097-8107, 2005.

30. Lee CW, Chuang LS, Kimura S, et al: RUNX3 functions as an oncogene in ovarian cancer. Gynecol Oncol 122: 410-417, 2011.

31. Yamamura Y, Lee WL, Inoue K, et al: RUNX3 cooperates with FoxO3a to induce apoptosis in gastric cancer cells. J Biol Chem 281: 5267-5276, 2006.

32. Yano T, Ito K, Fukamachi H, et al: The RUNX3 tumor suppressor upregulates Bim in gastric epithelial cells undergoing transforming growth factor beta-induced apoptosis. Mol Cell Biol 26: 4474-4488, 2006.

33. Inoue KI and Ito Y: Neuroblastoma cell proliferation is sensitive to changes in levels of RUNX1 and RUNX3 protein. Gene 28: 1379-1384, 2009. 